\title{
Large Scale Mood and Stress Self-Assessments on a Smartwatch
}

Katrin Hänsel

Queen Mary University of

London

London, United Kingdom

k.hansel@qmul.ac.uk

\section{Akram Alomainy}

Queen Mary University of

London

London, United Kingdom

a.alomainy@qmul.ac.uk

\section{Hamed Haddad}

Queen Mary University of

London

London, United Kingdom

hamed.haddadi@qmul.ac.uk

Permission to make digital or hard copies of part or all of this work for personal or

classroom use is granted without fee provided that copies are not made or distributed for profit or commercial advantage and that copies bear this notice and the full citation on the first page. Copyrights for third-party components of this work must be honored. For all other uses, contact the Owner/Author.

Copyright is held by the owner/author(s).

Ubicomp/ISWC'16 Adjunct, September 12-16, 2016, Heidelberg, Germany

ACM 978-1-4503-4462-3/16/09.

DOI: http://dx.doi.org/10.1145/2968219.2968305

\begin{abstract}
Modern sensing technology is becoming increasingly ubiquitous. Mobile phone sensing data has been used in research to address health and wellbeing; but in the last years, wearable technology became broadly available and popular. This opens new opportunity for health and wellbeing research in the wild. We will present an easy-to-use application to log current emotional states on a widely used smartwatch and collect additional, body sensing data to build a basis for new algorithms, interventions and technologysupported therapy around this data to promote emotional and mental well-being
\end{abstract}

\section{Author Keywords}

wearable sensing, stress, affective state

\section{ACM Classification Keywords}

H.5.m [Information interfaces and presentation (e.g., $\mathrm{HCl}$ )]: Miscellaneous; J.3 [Computer Applications]: Life And Medical Science - Consumer Health

\section{Introduction}

Stress and Emotional Well-being

Mental heath problems are a growing public health concern and can be seen as one of the main causes for diseases world wide [11]. Chronic stress and affective disorders can have a significant, negative influence on our well-being and 


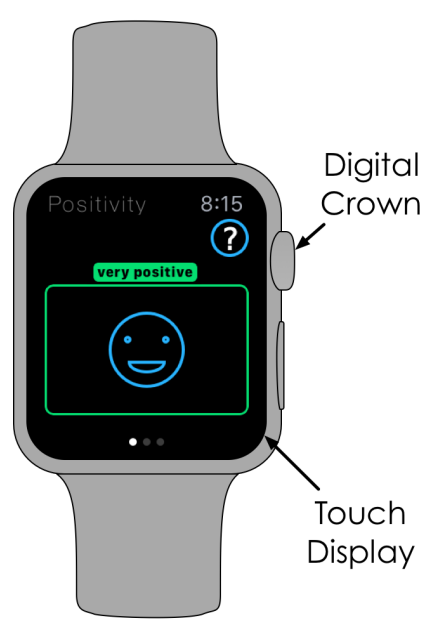

Figure 1: The Apple Watch UI: the Digital Crown wheel is used for scrolling through answer options; the touch display is used for swiping gestures and clicking the submit button. health. Knowledge about the user's current affective and emotional state and identification of critical states such as depression or stress can build the basis for new interventions, targeted therapies and prevention through early detection. Technology, and especially ubiquitous mobile and wearable devices, can support these strategies to target mental health and well-being.

\section{Mobile and Wearable Sensing Advances}

Advancing and miniaturising technology enabled current trends towards connected, smart, and highly sensor-equipped mobile and wearable devices. Especially, personal fitness trackers and bio-signal sensing smartwatches are popular amongst consumers and this trend is prognosed to continue [3]. This growing interest of consumers in their own health data and trends like the Quantified Self and Personal Informatics movement are drivers for new technology advances and products. Platform for personal health data storage, like Apple's HealthKit or Google's Health, not just allow eased data availability for consumers, but also provide new opportunities for health studies in the wild. There are already frameworks targeting these areas to ease the process of developing large scale research apps, like Apple's ResearchKit ${ }^{1}$ or ResearchStack ${ }^{2}$ for Android.

Opposing to mere mobile devices, wearables offer the advantage of the closeness to the body. Physiological signals like heart rate, skin conductance and blood pressure can be used to predict current emotional states and mood [5, 7]; but most of these studies have been conducted using expensive medical devices for targeted for experimental settings or specially designed devices, just available to a few. The utilisation of widely available consumer wearable devices, like the Apple Watch, allow for a broader user base

\footnotetext{
${ }^{1} \mathrm{http}: / /$ researchkit.org/

${ }^{2}$ http://researchstack.org/
}

and large-scale, in-the-wild data collection and interventions. But problems arise in terms of data reliability and with these uncertified and untested devices, which makes prior evaluation crucial.

Gathered and analysed sensing data can build the basis for new technology-driven intervention and prevention approaches. We will present a wearable application, based on the widely-used Apple Watch smartwatch, which eases the collection of mood experience samples and sensing data, such as heart rate, location, ambient noise, and physical activity. This application builds the basis for data-driven interventions and therapies.

\section{Related Work}

The computational power and sensor richness of mobile phones allows researchers to leverage these for detecting emotional and well-being states of users. Mobile phone

data, such as call/SMS/app usage, location can be used to infer current mood[4]. Other researchers have used mobile phone usage data to determine states of boredom [6]. The StudentLife project used mobile phone data of students and correlated it with academic performance and depression levels [12]. The Affective Diary used mobile phone usage data, photos and bluetooth to detect nearby people [10]; the provided diary functionality aimed to support reflection. EmotionSense is an Android app for social psychology experiments [8]. Audio samples during the day are used for speaker recognition and emotion classification based on speech prosody.

Related research has also focused on the utilisation of body-signals for inferring affective and psychological arousal states. Electrodermal activity and heart activity, such as variation of the beat intervals, have been widely used for affective state recognition [2]. 

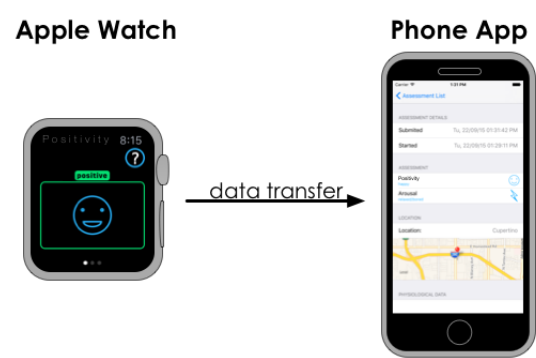

Collected Data: current mood - wrist movement ambient noise - Heart rate, workouts, and physical activi HealthKit
Collected Data: complex questionnaires for study workouts logged in Healthkit - location

Further Features: - users can review their emotional states

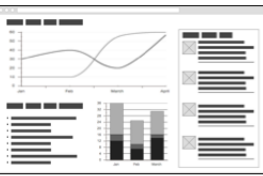

\section{Personalised Web} Dashboard:

- visualisations of own data - presentation of personalised recommendations
Figure 2: Overview of the envisioned application architecture

\section{Design of the App}

In this section, we present the design of a mobile phone and smartwatch application for collecting mood experience samples enriched with mobile/wearable sensing data. This data is the basis for evaluating the suitability of the Apple Watch as data provider and for developing future algorithms to infer current emotional levels based on the mobile and wearable sensing data. This can help to build future wellbeing applications and interventions designed around this data. Figure 2 shows an overview of the system we envision. It consists of a smartwatch and mobile app for data collection, a server component for storing the data and making it accessible for analysis by the research team. Additionally, a web-dashboard component is planned to enrich the user experience and data access. This dashboard will enable future studies and interventions around promotion of emotional well-being trough raising self-awareness and re-

\begin{tabular}{ll|ll}
\multicolumn{2}{c}{ Positivity } & \multicolumn{2}{c}{ Activeness } \\
\hline Description & Icon & \multicolumn{1}{c}{ Description } \\
\hline very positive & Icon \\
positive & & very excited/tense \\
neutral & -0 & tense \\
negative & neutral energy & relaxed/bored \\
very negative & -0 & very relaxed/bored \\
no answer & --- & no answer
\end{tabular}

Table 1: Icons and descriptions used for the self-assessments on the Apple Watch

flection. Since this component is in future planning, we here will focus on the smartwatch and mobile app design.

\section{The Apple Watch App}

The Apple Watch app is used to collect the mood experience samples and most of the sensing data. Users get multiple reminders a day to rate the current mood. We base these emotional self-assessments on a two-dimensional approach for affect classification - the Circumplex Model of Affect [9] which is a widely used model to quantify mood in a two-dimensional space: valence (the positivity) and psychological arousal (the activeness). During the selfassessments, the users are asked to rate their current emotion in two steps; the positivity and the activeness, both on a 5-point scale. They also have the option to not give an answer. An icon, representing the rated value and a text description have been used to depict the answer options. The choice for the use of smileys (positivity) and thunderbolts (arousal) has been based on the Self-Assessment Man- 


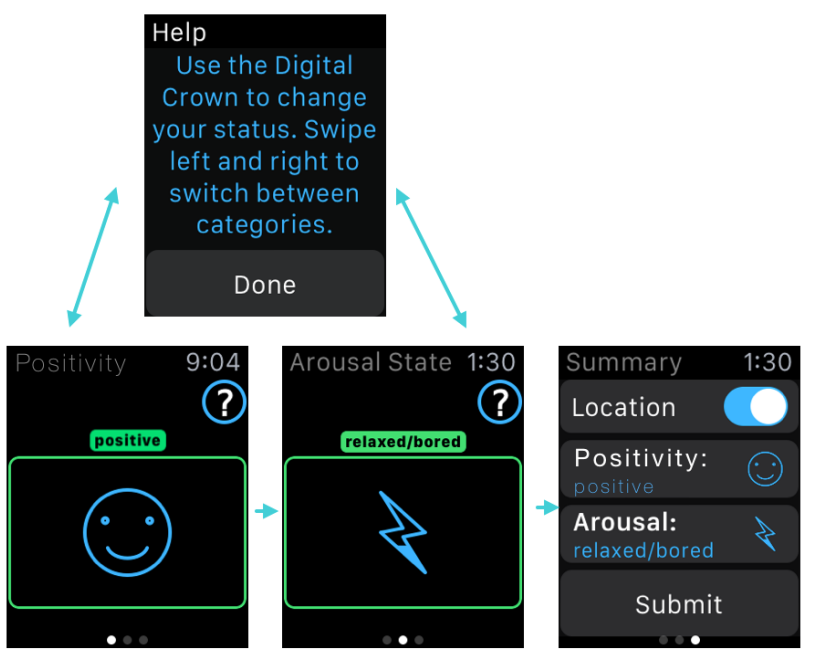

Figure 3: App Flow on the Apple Watch: The user can swipe through the 3 screen to rate the current affective state and submit on the final screen. A 'help' page offers instructions and examples of mood ratings for common affective states

nequin (SAM) scale [1] and varied/simplified to integrate in the app design. The options are presented in Table 1.

The Apple Watch offers special UI elements for interactions with the small device. The screen is touch sensitive; a button and digital crown offer further interactions. An example view of the app, buttons and the Digital Crown can be seen in Figure 1. We utilised these input mechanisms for the app interaction. The users can swipe through the app to switch between the following app screens: rate the positivity of the mood, rate the activeness of the mood, and the review/submit screen. Furthermore, the app offers a help screen with instructions on how to use the app and example positivity-activeness pairings for common moods. An overview of the app flow can be seen in Figure 3.

\section{The Phone App}

The watch app has a mobile phone companion, which offers the option of reviewing past self-assessments. This could offer a simple mechanism for reflection. We plan a more advanced option in form of an online dashboard in the future. Another purpose of the app is the delivery of complex questionnaires and study elements, which are not suitable to be delivered on a small watch screen. For a small test study, we used ResearchKit to develop questionnaires. We further plan to include mechanisms for context-aware reminders.

\section{Collected Sensing Data}

Additionally to the emotion self-assessments, we collect sensing data from the phone and watch. These include current location, heart rate, prior physical activity (steps and workouts), ambient noise and wrist movements from the watch accelerometer. The phone app also collects the data from the questionnaires.

\section{Future Direction}

In the near future, we plan a small scale study to evaluate the smartwatch app and the suitability of the collected data to infer current emotional and affective states with special focus on stress. Based on this, we will develop algorithms for automated emotion inference based on sensing data.

For the future, we envision the enhancement of the app system by a secure online storage unit, which provides easier data access and analysis for our research. We furthermore plan the extension with a web dashboard with personalised data visualisations and recommendations to enable reflection, support self-regulation and awareness. This platform can also be the basis for future data-driven interventions and therapies. 


\section{Conclusion}

We presented a smartwatch app for easy, daily mood selfassessments and sensing data collection. It could built the basis for future data-driven interventions, therapies and a tool for reflection and promotion of emotional well-being.

\section{REFERENCES}

1. Margaret M Bradley and Peter J Lang. 1994.

Measuring emotion: the Self-Assessment Manikin and the Semantic Differential. Journal Of Behavior Therapy And Experimental Psychiatry 25, 1 (March 1994), 49-59.

2. Rafael A Calvo and Sidney D'Mello. 2010. Affect Detection: an Interdisciplinary Review of Models, Methods, and Their Applications. IEEE Transactions on Affective Computing 1, 1 (Jan 2010), 18-37.

3. Tony Danova. 2015. The Wearables Report: Growth trends, consumer attitudes, and why smartwatches will dominate. (May 2015).

http://uk.businessinsider.com/ the-wearable-computing-market-report-2014-10

4. Robert LiKamWa, Yunxin Liu, Nicholas D Lane, and Lin Zhong. MoodScope. In Proceeding of the 11th annual international onference on Mobile Systems,

Applications, and Services (MobiSys'13).

5. Daniel McDuff, Amy Karlson, Ashish Kapoor, Asta Roseway, and Mary Czerwinski. AffectAura: an intelligent system for emotional memory. In Proceedings of the SIGCHI Conference on Human Factors in Computing Systems (CHl'12).

6. Martin Pielot, Tilman Dingler, Jose San Pedro, and Nuria Oliver. When Attention Is Not Scarce - Detecting Boredom From Mobile Phone Usage. In Proceedings of the 2015 ACM International Joint Conference on Pervasive and Ubiquitous Computing (UbiComp'15).
7. M T Quazi, S C Mukhopadhyay, N K Suryadevara, and Y M Huang. Towards the smart sensors based human emotion recognition. In 2012 IEEE International Instrumentation and Measurement Technology Conference (I2MTC'12).

8. Kiran K Rachuri, Mirco Musolesi, Cecilia Mascolo, Peter J Rentfrow, Chris Longworth, and Andrius Aucinas. 2010. EmotionSense: a mobile phones based adaptive platform for experimental social psychology research. In Proceedings of the 12th ACM international conference on Ubiquitous Computing (UbiComp'10).

9. James A Russell. 1980. A Circumplex Model of Affect. Journal of Personality and Social Psychology 39, 6 (1980), 1161-1178.

10. Anna Ståhl, Kristina Höök, Martin Svensson, Alex S. Taylor, and Marco Combetto. 2009. Experiencing the Affective Diary. Personal Ubiquitous Comput. 13, 5 (June 2009), 365-378.

11. Theo Vos, et al. 2015. Global, regional, and national incidence, prevalence, and years lived with disability for 301 acute and chronic diseases and injuries in 188 countries, 1990-2013: a systematic analysis for the Global Burden of Disease Study. The Lancet 386, 9995 (Aug. 2015), 743-800.

12. Rui Wang, Fanglin Chen, Zhenyu Chen, Tianxing Li, Gabriella Harari, Stefanie Tignor, Xia Zhou, Dror Ben-Zeev, and Andrew T Campbell. StudentLife: Assessing Mental Health, Academic Performance and Behavioral Trends of College Students Using Smartphones. In Proceedings of the 2014 ACM International Joint Conference on Pervasive and Ubiquitous Computing (UbiComp'14). 